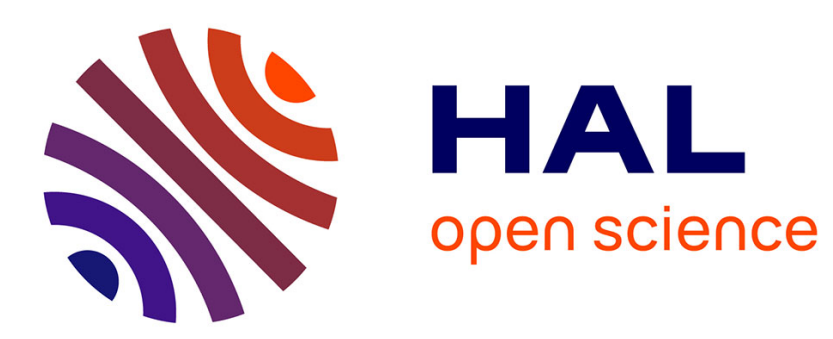

\title{
From DEL to EDL: exploring the power of converse events
}

\author{
Guillaume Aucher, Andreas Herzig
}

\section{To cite this version:}

Guillaume Aucher, Andreas Herzig. From DEL to EDL: exploring the power of converse events. [Research Report] IRIT: Institut de recherche en informatique de Toulouse. 2007. hal-03516649

\section{HAL Id: hal-03516649 \\ https://hal.science/hal-03516649}

Submitted on 10 Jan 2022

HAL is a multi-disciplinary open access archive for the deposit and dissemination of scientific research documents, whether they are published or not. The documents may come from teaching and research institutions in France or abroad, or from public or private research centers.
L'archive ouverte pluridisciplinaire HAL, est destinée au dépôt et à la diffusion de documents scientifiques de niveau recherche, publiés ou non, émanant des établissements d'enseignement et de recherche français ou étrangers, des laboratoires publics ou privés. 


\title{
From DEL to EDL: Exploring the Power of Converse Events
}

\author{
Guillaume Aucher, Andreas Herzig \\ University of Otago (NZ) - University Paul Sabatier (F) \\ IRIT, Université Paul Sabatier, 31062 Toulouse Cedex (F) \\ aucher@irit.fr, herzig@irit.fr
}

\begin{abstract}
Dynamic epistemic logic (DEL) as viewed by Baltag et col. and propositional dynamic logic (PDL) offer different semantics of events. On the one hand, DEL adds dynamics to epistemic logic by introducing so-called epistemic action models as syntactic objects into the language. On the other hand, PDL has instead transition relations between possible worlds. This last approach allows to easily introduce converse events. We add epistemics to this, and call the resulting logic epistemic dynamic logic (EDL). We show that DEL can be translated into EDL thanks to this use of the converse operator: this device enables us to translate the structure of the action (or event) model directly within a particular axiomatization of EDL, without having to refer to a particular epistemic action (event) model in the language (as done in DEL). It follows that EDL is more expressive and general than DEL.
\end{abstract}

Note 1. This report is an extended version with proofs of a paper that has been published (without proofs) in the proceedings of ECSQARU'07.

\section{Introduction}

Aim: reason about perception of events. To account for various modes of perception of events is the aim of a family of formal systems called dynamic epistemic logics. These systems were proposed in a series of publications most prominently by Plaza, Baltag, Gerbrandy, van Benthem, van Ditmarsch, and Kooi [10, 7, 6, $14,16,17]$. Dynamic epistemic logics add dynamics to Hintikka's epistemic logic via transformations of its models.

The focus of dynamic epistemic logics is on particular events that are called updates. Updates can be seen as announcements made to the agents. The simplest case of updates are public announcements à la Plaza [10]; when the input is propositional such announcements correspond to AGM expansion operations [1]. Another example are group announcements à la Gerbrandy [6,7]. Note that DEL-updates differ from Katsuno-Mendelzon-like updates as studied in the AI literature $[9]$.

In $[2,4,3]$ and elsewhere, Baltag et col. proposed a dynamic epistemic logic that was very influential. We refer to it in this paper by the term DEL. It has 
been shown that their account subsumes all other dynamic epistemic logics, justifying our acronym. The semantics of DEL is based on two kinds of models: a static model $M^{s}$ (called state model by Baltag) and a (finite) dynamic model $M^{d}$ (called epistemic action model by Baltag). $M^{s}$ models the actual world and the agents' beliefs about it, and is nothing but a good old epistemic model à la Hintikka. $M^{d}$ models the actual event taking place and the agents' beliefs about it. An agent's beliefs can be incomplete (event $a$ occurred, but agent cannot distinguish occurrence of $a$ from occurrence of $a^{\prime}$ ) and even unsound ( $a$ occurred, but agent wrongly perceived some $\left.a^{\prime}\right) . M^{s}$ and $M^{d}$ are then combined by a restricted product construction which defines the situation after the actual event took place, viz. the resulting actual world, and the agents' beliefs about it.

Semantics of events: products vs. accessibility relations. Naturally, we would be interested to express in DEL that an event a occurred, viz to give semantics to the converse event $a^{-}$within the framework of DEL. It is not clear how this should work precisely. The only approach we are aware of is that of Yap [19] who fails to get a complete characterization.

On the other hand, in PDL, events are interpreted as transition relations on possible worlds, and not as restricted products of models as in DEL. Converse events $a^{-}$can then easily be interpreted by inverting the accessibility relation associated to $a$. The resulting logic is called the tense extension of PDL.

To this we then add an epistemic operator. We call (tensed) Epistemic Dynamic Logic EDL the combination of epistemic logic and PDL with converse. ${ }^{1}$

A semantics in terms of transition relations is more flexible than DEL's product semantics: we have more options concerning the interaction between events and beliefs. Our main contribution here is to account for this delicate relationship by means of constraints on the respective accessibility relations: a no-forgetting and a no-learning constraint, and a constraint of epistemic determinism.

Translating DEL into EDL. To demonstrate the power of our approach we provide a translation from DEL to EDL: we express the structure of a DEL dynamic model $M^{d}$ by a nonlogical theory $\Gamma\left(M^{d}\right)$ of EDL, and prove that any formula $\varphi$ is valid in DEL if and only if it is a logical consequence of $\Gamma\left(M^{d}\right)$ in EDL.

So, unlike DEL, we avoid to refer to a semantical structure (viz. the DEL dynamic model $M^{d}$ ) in the very definition of the language. Encoding the structure of a DEL dynamic model $M^{d}$ by a nonlogical theory $\Gamma\left(M^{d}\right)$ of EDL is done thanks to converse events. For example $[a] B_{i}\left(\left\langle a^{-}\right\rangle \top \vee\left\langle b^{-}\right\rangle \top\right)$ expresses that agent $i$ perceives the occurrence of $a$ as that of either $a$ or $b$.

${ }^{1}$ EDL is related to Segerberg's Doxastic Dynamic Logic DDL $[12,13]$. Up to now research on DDL focussed mainly on its relation with the AGM theory of belief revision, and studied particular events of the form $+\varphi$ (expansion by $\varphi$ ), $* \varphi$ (revision by $\varphi$ ), and $-\varphi$ (contraction by $\varphi$ ). EDL and DDL coincides in what concerns propositional announcements. 
Organization of the paper. This paper is organized as follows. In section 2 we introduce a language of belief, events and converse events. In section 3 we provide a semantics for that language, and define our logic EDL. In section 4 we give Baltag's restricted product semantics for the fragment of the language without converse, and define his logic DEL. In section 5 we associate a theory $\Gamma\left(M^{d}\right)$ to each dynamic model $M^{d}$, and prove that the consequences of $\Gamma\left(M^{d}\right)$ in EDL match the DEL-validities. This suggests that EDL is more expressive and general than DEL, and we will concentrate on that point to conclude in section 6 .

\section{The Languages}

We suppose given sets of propositional symbols $P R O P=\{p, q, \ldots\}$, agent symbols $A G T=\{i, j, \ldots\}$, and event symbols $E V T=\{a, b, \ldots\}$. All these sets may be infinite (while in DEL $A G T$ and $E V T$ have to be finite). From these ingredients the multi-modal language is built classically as follows:

$$
\varphi:=\perp|p| \neg \varphi\left|\varphi \wedge \varphi^{\prime}\right| B_{i} \varphi|[a] \varphi|\left[a^{-}\right] \varphi, p \in P R O P, i \in A G T, a \in E V T
$$

The formula $B_{i} \varphi$ reads "agent $i$ believes that $\varphi$ ". $[a] \varphi$ reads " $\varphi$ holds after every possible occurrence of event $a$ ". $\left[a^{-}\right] \varphi$ reads " $\varphi$ held before $a$ ". The dual modal operators $\hat{B}_{i},\langle a\rangle$, and $\left\langle a^{-}\right\rangle$are defined in the usual way: $\hat{B}_{i} \varphi$ abbreviates $\neg B_{i} \neg \varphi ;\langle a\rangle \varphi$ abbreviates $\neg[a] \neg \varphi ;\left\langle a^{-}\right\rangle \varphi$ abbreviates $\neg\left[a^{-}\right] \neg \varphi$.

The language $\mathcal{L}_{\text {EDL }}$ of EDL is the entire language. The language $\mathcal{L}_{\mathrm{DEL}}$ of DEL is the set of those formulas of $\mathcal{L}_{\mathrm{EDL}}$ that do not contain the converse operator $\left[a^{-}\right]$. Finally, the epistemic language $\mathcal{L}_{E L}$ is the set of those formulas of $\mathcal{L}_{\mathrm{EDL}}$ that do not contain any dynamic operator, i.e. built from PROP, the Boolean operators and the $B_{i}$ operators alone. For example $[a] B_{i}\left[a^{-}\right] \perp$ is an $\mathcal{L}_{\mathrm{EDL}}$-formula (that is not in $\mathcal{L}_{\mathrm{DEL}}$ ).

\section{EDL: Epistemic Dynamic Logic with Converse}

When designing models of events and beliefs the central issue is to account for the interplay of these two concepts. In our PDL-based semantics this is done by means of constraints on the respective accessibility relations. These will ensure what we call no-forgetting, no-learning and epistemic determinism.

\subsection{Semantics}

EDL-models are of the form

$$
M=\left\langle W, V,\left\{\mathcal{A}_{a}\right\}_{a \in E V T},\left\{\mathcal{B}_{i}\right\}_{i \in A G T}\right\rangle
$$

where $W$ is a set of possible worlds, $V: P R O P \longrightarrow 2^{W}$ a valuation, and the $\mathcal{A}_{a} \subseteq W \times W$ and $\mathcal{B}_{i} \subseteq W \times W$ are accessibility relations on $W$. The relation $\mathcal{A}_{a}^{-1}$ is the inverse of $\mathcal{A}_{a}$. We sometimes view accessibility relations as mappings 
from worlds to sets of worlds, and write for example $\mathcal{A}_{a}^{-1}(w)=\{v:\langle w, v\rangle \in$ $\left.\mathcal{A}_{a}^{-1}\right\}=\left\{v:\langle v, w\rangle \in \mathcal{A}_{a}\right\}$.

We suppose that EDL-models satisfy the following constraints of no forgetting, no learning and epistemic determinism:

(nf) If $v\left(\mathcal{A}_{a} \circ \mathcal{B}_{i} \circ \mathcal{A}_{b}^{-1}\right) v^{\prime}$ then $v \mathcal{B}_{i} v^{\prime}$.

(nl) If $\left(\mathcal{A}_{a} \circ \mathcal{B}_{i} \circ \mathcal{A}_{b}^{-1}\right)(v) \neq \emptyset$ then $\left(\mathcal{B}_{i} \circ \mathcal{A}_{b}\right)(v) \subseteq\left(\mathcal{A}_{a} \circ \mathcal{B}_{i}\right)(v)$.

(ed) If $w_{1}, w_{2} \in \mathcal{A}_{a}(v)$ then $\mathcal{B}_{i}\left(w_{1}\right)=\mathcal{B}_{i}\left(w_{2}\right)$.

To understand the no-forgetting principle, also known as perfect recall [5], suppose that $w$ results from the occurrence of event $a$ in world $v$; if in world $w$, the world $w^{\prime}$ is an alternative for agent $i$, and $w^{\prime}$ results from event $b$ in a world $v^{\prime}$, then $v^{\prime}$ was already possible for agent $i$ in the world $v$ (see figure below).

To understand the principle no-learning, also known as no miracle [15], assume that agent $i$ perceive the occurrence of $a$ as that of $b_{1}, b_{2} \ldots$ or $b_{n}$. Then, informally, the no-learning principle says that all such alternatives resulting from occurrence of $b_{1}, b_{2}, \ldots, b_{n}$ in $i$ 's alternatives before $a$ are indeed alternatives after $a$. Formally, assume that agent $i$ perceives $b$ as a possible alternative of $a$ (i.e. $\left(\mathcal{A}_{a} \circ \mathcal{B}_{i} \circ \mathcal{A}_{b}^{-1}\right)(v) \neq \emptyset$ ). If at $v$ world $w^{\prime}$ was a possible outcome of event $b$ for $i$, then $w^{\prime}$ is possible for $i$ at some $w \in \mathcal{A}_{a}(v)$ (see figure below).

Finally, the epistemic determinism principle says that an agent's epistemic state after an event does not depend on the particular nondeterministic outcome. Formally, suppose we have $v \mathcal{A}_{a} w_{1}$ and $v \mathcal{A}_{a} w_{2}$. Then (ed) forces that the epistemic states at $w_{1}$ and $w_{2}$ are identical: $\mathcal{B}_{i}\left(w_{1}\right)=\mathcal{B}_{i}\left(w_{2}\right)$ (see figure below). This follows from our hypothesis that events are feedback-free (also known as uninformative events [8]): the agents cannot distinguish between their different nondeterministic outcomes. These are events of which the agents only learn their occurrence, but not their outcomes. Both public and private announcements are examples of feedback-free events. Another example is the event of tossing a coin without checking the result. An example of an event that is not feedback-free is agent $i$ 's event of testing if formula $\varphi$ is true: beyond the mere occurrence of the test, $i$ also learns about its outcome, i.e. after the test $i$ knows whether $\varphi$ is true or not. Thus the no-learning constraint is violated. Another example of a non-feedback-free event is that of tossing a coin and looking at it: here the epistemic determinism constraint is violated.

Truth of a formula $\varphi$ in a world $w$ of a model $M$ is noted $M, w \models \varphi$ and is defined as usual:

$$
\begin{gathered}
M, w \models p \text { iff } w \in V(p) \\
M, w \models \neg \varphi \text { iff it is not the case that } M, w \models \varphi \\
M, w \models \varphi \wedge \varphi^{\prime} \text { iff } M, w \models \varphi \text { and } M, w \models \varphi^{\prime} \\
M, v \models B_{i} \varphi \text { iff } M, v^{\prime} \models \varphi \text { for every } w^{\prime} \in \mathcal{B}_{i}(v) \\
M, v \models[a] \varphi \text { iff } M, w \models \varphi \text { for every } w \in \mathcal{A}_{a}(v) \\
M, w \models\left[a^{-}\right] \varphi \text { iff } M, v \models \varphi \text { for every } v \in \mathcal{A}_{a}^{-1}(w)
\end{gathered}
$$



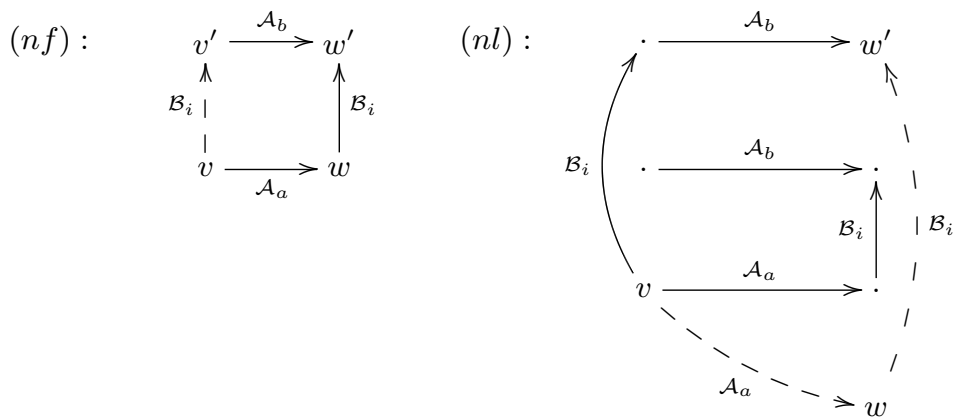

$(e d)$ :

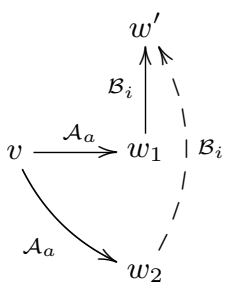

Truth of $\varphi$ in a EDL-model $M$ is noted $M \models \varphi$ and is defined as: $M, w \models \varphi$ for every $w \in W$. Let $\Gamma$ be a set of $\mathcal{L}_{\mathrm{EDL}}$-formulas. The (global) consequence relation is defined by:

$\Gamma \models$ EDL $\varphi$ iff for every EDL-model $M$, if $M \models \psi$ for every $\psi \in \Gamma$ then $M \models \varphi$.

For example we have

and

$$
\left\{[b] \varphi,\langle a\rangle B_{i}\left\langle b^{-}\right\rangle \top\right\} \mid=_{\mathrm{EDL}}[a] B_{i} \varphi
$$

$$
\models_{\mathrm{EDL}}\left(B_{i}[b] \varphi \wedge\langle a\rangle B_{i}\left\langle b^{-}\right\rangle \top\right) \rightarrow[a] B_{i} \varphi .\left(^{*}\right)
$$

Consider $\varphi=\perp$ in $(*): B_{i}[b] \perp$ means that perception of event $b$ was unexpected by agent $i$, while $\langle a\rangle B_{i}\left\langle b^{-}\right\rangle \top$ means that $i$ actually perceives $a$ as $b$. By our no-forgetting constraint it follows that $[a] B_{i} \perp$ In fact, one would like to avoid agents getting inconsistent: in such situations some sort of belief revision should take place. We do not investigate this further here, and leave it to future work to augment EDL by belief revision mechanisms.

\subsection{Completeness}

The axiomatics of EDL is made up of the principles of multi-modal logic $\mathrm{K}$ for all the modal operators $B_{i},[a]$ et $\left[a^{-}\right]$, plus the axioms $\left(\mathrm{Conv}_{1}\right),\left(\mathrm{Conv}_{2}\right),(\mathrm{NF})$ et (NL) below:

$$
\begin{array}{ll}
\left(\mathrm{Conv}_{1}\right) & \vdash_{\mathrm{EDL}} \varphi \rightarrow[a]\left\langle a^{-}\right\rangle \varphi \\
\left(\mathrm{Conv}_{2}\right) & \vdash_{\mathrm{EDL}} \varphi \rightarrow\left[a^{-}\right]\langle a\rangle \varphi \\
(\mathrm{NF}) & \vdash_{\mathrm{EDL}} B_{i} \varphi \rightarrow[a] B_{i}\left[b^{-}\right] \varphi \\
(\mathrm{NL}) & \vdash_{\mathrm{EDL}}\langle a\rangle \hat{B}_{i}\left\langle b^{-}\right\rangle \top \rightarrow\left([a] B_{i} \varphi \rightarrow B_{i}[b] \varphi\right) \\
(\mathrm{ED}) & \vdash_{\mathrm{EDL}}\langle a\rangle B_{i} \varphi \rightarrow[a] B_{i} \varphi
\end{array}
$$

(Conv1) and (Conv2) are the standard conversion axioms of tense logic and converse PDL. (NF), (NL) and (ED) respectively axiomatize no forgetting, no learning and epistemic determinism. 
We write $\Gamma \vdash_{\text {EDL }} \varphi$ when $\varphi$ is provable from the set of formulas $\Gamma$ in this axiomatics.

EDL is strongly complete:

Proposition 1. For every set of $\mathcal{L}_{E D L}$-formulas $\Gamma$ and $\mathcal{L}_{E D L}$-formula $\varphi$,

$$
\Gamma \models_{E D L} \varphi \text { if and only if } \Gamma \vdash_{E D L} \varphi \text {. }
$$

Proof. The proof follows from Sahlqvist's theorem [11]: all our axioms (NF), $(\mathrm{NL}),(\mathrm{ED})$ are of the required form, and match the respective constraints (nf), (nl), (ed).

\subsection{Extensions of the Basic Logic}

We are going to study how our constraint (nl) evolves when we add other constraints on the epistemic accessibility relation $\mathcal{B}_{i}$, as it is often done in epistemic logic.

Introspective Belief In the literature, the notion of belief is often supposed to satisfy positive and negative introspection. That is to say, the axioms of transitivity (4) and of euclidianity (5) are valid: $B_{i} \varphi \rightarrow B_{i} B_{i} \varphi(4)$ and $\neg B_{i} \varphi \rightarrow$ $B_{i} \neg B_{i} \varphi(5)$. Semantically, in that case every $\mathcal{B}_{i}$ satisfies:

$(45)$ if $w \mathcal{B}_{i} w^{\prime}$ then $\mathcal{B}_{i}(w)=\mathcal{B}_{i}\left(w^{\prime}\right)$

Proposition 2. Conditions (45), (nl), (nf) and (ed) are equivalent to conditions (45), $\left(n l^{i}\right)$, (nf) and (ed) where

$\left(n l^{i}\right) \quad \mathcal{B}_{i} \circ \mathcal{A}_{a}^{-1} \circ \mathcal{B}_{i} \circ \mathcal{A}_{a} \subseteq \mathcal{B}_{i}$

This proposition tells us that under $(45),(\mathrm{nl})$ simplifies to $\left(\mathrm{nl}^{i}\right)$. The axiom corresponding with $\left(\mathrm{nl}^{i}\right)$ is $B_{i} \varphi \rightarrow B_{i}\left[a^{-}\right] B_{i}[a] \varphi$.

Knowledge In the literature, the notion of knowledge is often supposed to satisfy positive and negative introspection but also reflexivity $(\mathrm{T}): B_{i} \varphi \rightarrow \varphi$ (T). Semantically, (T) corresponds to

(t) $\quad w \mathcal{B}_{i} w$

One can then show that with this extra condition $\mathcal{B}_{i}$ is an equivalence relation.

Proposition 3. Conditions (45), (t), (nl), (nf), (ed) are equivalent to (45), (t), $\left(n l^{t}\right)$, (nf), (ed) where

$\left(n l^{t}\right) \quad \mathcal{A}_{a}^{-1} \circ \mathcal{B}_{i} \circ \mathcal{A}_{a} \subseteq \mathcal{B}_{i}$

Proposition 3 tells us that under (45) and (t), (nl) simplifies to $\left(\mathrm{nl}^{t}\right)$. The axiom corresponding with $\left(\mathrm{nl}^{t}\right)$ is $B_{i} \varphi \rightarrow\left[a^{-}\right] B_{i}[a] \varphi$ and is somewhat symmetric w.r.t. axiom (NF). 


\section{DEL: Static Models, Dynamic Models, and their Products}

We here present the star-free version of Baltag's dynamic epistemic logic DEL $[4,3]$.

\subsection{Semantics}

- Static models are just models of the form $M^{s}=\left\langle W, V,\left\{\stackrel{s}{\longrightarrow}_{i}\right\}_{i \in A G T}\right\rangle$, where $W$ is an arbitrary set, $V: P R O P \longrightarrow 2^{W}$ a valuation and the $\stackrel{s}{\longrightarrow} \subseteq$ $W \times W$ are accessibility relations on $W$.

- Dynamic models are of the form $M^{d}=\left\langle E V T\right.$, Pre, $\left.\left\{\stackrel{d}{\longrightarrow}_{i}\right\}_{i \in A G T}\right\rangle$, where Pre $: E V T \longrightarrow \mathcal{L}_{E L}$ is a precondition function associating epistemic formulas to events, and the $\stackrel{d}{\longrightarrow} \subseteq E V T \times E V T$ are accessibility relations on $E V T$.

Intuitive interpretation. Informally, $\operatorname{Pre}(a)$ is the precondition that a world must fulfill so that the event $a$ can take place in this world. For example $\operatorname{Pre}(a)=\top$ means that action $a$ can take place in any world. When we have $\stackrel{d}{\longrightarrow}(a)=\{b\}$ then the occurrence of $a$ is perceived by agent $i$ as the occurrence of $b$; when $\stackrel{d}{\longrightarrow}$ i $(a)=\left\{b_{1}, b_{2}\right\}$ then the occurrence of $a$ is perceived by agent $i$ indistinguishably as the occurrence of $b_{1}$ or $b_{2}$; etc.

We recall that the set $E V T$ is the set of atomic events. In DEL it is supposed to be finite. Moreover, every $\stackrel{d}{\longrightarrow}_{i}$ is supposed to be serial: for every $a \in E V T$ there is at least one $b \in E V T$ such that $a \stackrel{d}{\longrightarrow} b$.

Remark 1. The basic logic DEL does not validate introspective principles. DEL can be extended as usual such that for every $i \in A G T$, the $\stackrel{s}{\longrightarrow}_{i}$ and the $\stackrel{d}{\longrightarrow}_{i}$ are transitive and Euclidian.

- Product construction. Given $M^{s}=\left\langle W, V,\left\{\stackrel{s}{\longrightarrow}_{i}\right\}_{i \in A G T}\right\rangle$ and $M^{d}=$ $\left\langle E V T\right.$, Pre, $\left.\{\stackrel{d}{\longrightarrow}\}_{i \in E V T}\right\rangle$, their product $M^{s} \otimes M^{d}$ is a static model describing the situation after the event described by $M^{d}$ occurred in $M^{s}$ :

$$
M^{s} \otimes M^{d}=\left\langle W^{\prime}, V^{\prime},\left\{\stackrel{s}{\longrightarrow}_{i}^{\prime}\right\}_{i \in A G T}\right\rangle
$$

where the new set of possible worlds is $W^{\prime}=\left\{\langle w, a\rangle: M^{s}, w \models \operatorname{Pre}(a)\right\}$, the new valuation is $V^{\prime}(p)=\{\langle w, a\rangle: w \in V(p)\}$, and the new static accessibility relation is defined by

$$
\left\langle w_{1}, a_{1}\right\rangle \stackrel{s}{\longrightarrow}_{i}^{\prime}\left\langle w_{2}, a_{2}\right\rangle \text { iff } w_{1} \stackrel{s}{\longrightarrow}_{i} w_{2} \text { and } a_{1} \stackrel{d}{\longrightarrow}_{i} a_{2} .
$$

- While the truth condition for the epistemic operator is just as in Hintikka's epistemic logic and in EDL, the product construction gives a semantics to the $[a]$ operator which is quite different from that of PDL and EDL: 


$$
M^{s}, w \models[a] \varphi \text { iff } M^{s}, w \models \operatorname{Pre}(a) \text { implies } M^{s} \otimes M^{d},\langle w, a\rangle \models \varphi
$$

Finally, validity of $\varphi$ in DEL (noted $\models_{\text {DEL }} \varphi$ ) is defined as usual as truth in every world of every DEL-model. Note that validity means validity w.r.t. a fixed dynamic model $M^{d}$.

Remark 2. The truth condition for the dynamic operator highlights that DEL is a dynamic extension of epistemic logic, while EDL is an epistemic extension of PDL.

\subsection{Completeness}

Suppose given a dynamic model $M^{d}$. The axiomatics of DEL is made of the principles of the multi-modal logic $\mathrm{K}$ for the modal operators $B_{i}$ and $[a]$, together with the following axioms $[4,3]$.

$$
\begin{aligned}
& \vdash_{\text {DEL }}[a] p \leftrightarrow(\operatorname{Pre}(a) \rightarrow p) \\
& \vdash_{\mathrm{DEL}}[a] \neg \varphi \leftrightarrow(\operatorname{Pre}(a) \rightarrow \neg[a] \varphi) \\
& \vdash_{\mathrm{DEL}}[a] B_{i} \varphi \leftrightarrow\left(\operatorname{Pre}(a) \rightarrow B_{i}\left[b_{1}\right] \varphi \wedge \ldots \wedge B_{i}\left[b_{n}\right] \varphi\right) \\
& \text { where } b_{1}, \ldots, b_{n} \text { is the list of all } b \text { such that } a \stackrel{d}{\longrightarrow} i \text {. }
\end{aligned}
$$

We note $\vdash_{\text {DEL }} \varphi$ when $\varphi$ is provable from these principles. Note that this axiomatization depends on a particular dynamic model $M^{d}$.

For example for every dynamic model $M^{d}$ where $\operatorname{Pre}(a)=\top, \operatorname{Pre}(b)=p$, and $\stackrel{d}{\longrightarrow}(a)=\{b\}$ we obtain $\vdash_{\text {DEL }}[a] B_{i} p$. Indeed, $\vdash_{\text {DEL }}[a] B_{i} p \leftrightarrow\left(\operatorname{Pre}(a) \rightarrow B_{i}[b] p\right)$ and $\vdash_{\text {DEL }} B_{i}[b] p$ because $\vdash_{\text {DEL }}[b] p$.

\section{From DEL to EDL}

In this section we show that DEL can be embedded into EDL. We do that by building a particular EDL-theory that encode syntactically the structure of a given DEL dynamic model $M^{d}$.

Definition 1. Let $M^{d}=\left\langle E V T\right.$, Pre, $\left.\left\{\stackrel{d}{\longrightarrow}_{i}\right\}_{i \in A G T}\right\rangle$ be a dynamic model. The set of formulas $\Gamma\left(M^{d}\right)$ associated to $M^{d}$ ('the theory of $M^{d}$ ') is made up of the following non-logical axioms:

(1) $p \rightarrow[a] p$ and $\neg p \rightarrow[a] \neg p$, for every $a \in E V T$ and $p \in P R O P$;

(2) $\langle a\rangle T \leftrightarrow \operatorname{Pre}(a)$, for every $a \in E V T$;

(3) $[a] B_{i}\left(\left\langle b_{1}^{-}\right\rangle \top \vee \ldots \vee\left\langle b_{n}^{-}\right\rangle \top\right)$, where $b_{1}, \ldots, b_{n}$ is the list of all $b$ such that $a \stackrel{d}{\longrightarrow} i$

(4) $\hat{B}_{i} \operatorname{Pre}(b) \rightarrow[a] \hat{B}_{i}\left\langle b^{-}\right\rangle \top$, for every $\langle a, b\rangle \in \stackrel{d}{\longrightarrow}$.

Note that $\Gamma\left(M^{d}\right)$ is finite because in EDL both the set of events EVT and the set of agents $A G T$ are finite. 
Axiom 1 encodes the fact that events do not change propositional facts of the world where they are performed (cf the definition of $V^{\prime}(p)$ in Section 4.1). Axiom 2 encodes the fact that an event $a$ can occur in a world iff this world satisfies the precondition of event $a$ (cf the definition of $W^{\prime}$ in Section 4.1). Axiom 3 encodes the modal structure of the dynamic model. Axiom 4 encodes the definition of $\stackrel{s}{\longrightarrow}{ }_{i}^{\prime}($ cf Section 4.1).

Example 1. Consider that $A G T=\{A ; B\}$ and $P R O P=\{p\}$. In the figure below is represented the dynamic models $M_{1}^{d}$ and $M_{2}^{d}$ corresponding respectively to the public announcement of $\varphi$ and the private announcement of $\varphi$, where $\varphi \in \mathcal{L}_{E L}$. Here, $\operatorname{Pre}(a)=\varphi$ in both models and $\operatorname{Pre}(b)=\top$.

Public announcement of $\varphi: \quad$ Private announcement of $\varphi$ to A:
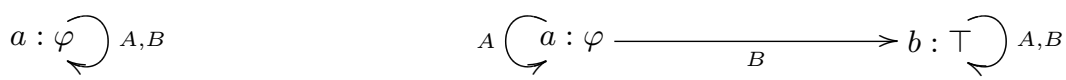

Applying Definition 1, we get

$\Gamma\left(M_{1}^{d}\right):=\left\{p \rightarrow[a] p\right.$ and $\neg p \rightarrow[a] \neg p ;\langle a\rangle \top \leftrightarrow \varphi ;[a] B_{A}\left(\left\langle a^{-}\right\rangle \top\right) ;$ $\left.\left.[a] B_{B}\left(\left\langle a^{-}\right\rangle \top\right) ; \hat{B_{A}} \varphi \rightarrow[a] \hat{B_{A}}\left\langle a^{-}\right\rangle \top\right\} ; \hat{B_{B}} \varphi \rightarrow[a] \hat{B_{B}}\left\langle a^{-}\right\rangle \top\right\}$

and

$\Gamma\left(M_{2}^{d}\right):=\{p \rightarrow[a] p$ and $\neg p \rightarrow[a] \neg p ; p \rightarrow[b] p$ and $\neg p \rightarrow[b] \neg p ;\langle a\rangle \top \leftrightarrow \varphi$ $;\langle b\rangle \top \leftrightarrow \top ;[a] B_{A}\left\langle a^{-}\right\rangle \top ;[a] B_{B}\left\langle b^{-}\right\rangle \top ;[b] B_{A}\left\langle b^{-}\right\rangle \top ;[b] B_{B}\left\langle b^{-}\right\rangle \top ; \hat{B_{A}} \varphi \rightarrow$ $\left.[a] \hat{B_{A}}\left\langle a^{-}\right\rangle \top ; \hat{B_{A}} \top \rightarrow[b] \hat{B_{A}}\left\langle b^{-}\right\rangle \top ; \hat{B_{B}} \top \rightarrow[a] \hat{B_{B}}\left\langle b^{-}\right\rangle \top ; \hat{B_{B}} \top \rightarrow[b] \hat{B_{B}}\left\langle b^{-}\right\rangle \top\right\}$

It turns out that the axiom of determinism is a logical consequence of $\Gamma\left(M^{d}\right)$ in EDL . This is comforting because the axiom of determinism is indeed valid in DEL .

Lemma 1. For every $\mathcal{L}_{D E L}$-formula $\varphi$ we have $\Gamma\left(M^{d}\right) \models_{E D L}\langle a\rangle \varphi \rightarrow[a] \varphi$.

Thanks to this lemma, we can now prove that for every formula $\varphi$ of the language $\mathcal{L}_{\mathrm{DEL}}, \models_{\mathrm{DEL}} \varphi$ if and only if $\Gamma\left(M^{d}\right) \models_{\mathrm{EDL}} \varphi$. We first prove two lemmas.

Lemma 2. Let $M^{d}$ be a DEL dynamic model, and let $\psi$ be a formula from $\mathcal{L}_{D E L}$. If $\forall{ }_{D E L} \psi$ then $\Gamma\left(M^{d}\right) \forall_{E D L} \psi$.

Lemma 3. Let $M^{d}$ be a DEL dynamic model, and let $\psi$ be a formula from $\mathcal{L}_{D E L}$. If $\models_{D E L} \psi$ then $\Gamma\left(M^{d}\right) \models_{E D L} \psi$.

Putting these two results together we obtain the following key result: 
Theorem 1. Let $M^{d}$ be a DEL dynamic model. Let $\psi$ be a formula from $\mathcal{L}_{D E L}$. Then

$$
\models_{D E L} \varphi \quad \text { iff } \Gamma\left(M^{d}\right) \models_{E D L} \varphi
$$

It follows that

$$
\vdash_{\mathrm{DEL}} \varphi \quad \text { iff } \Gamma\left(M^{d}\right) \vdash_{\mathrm{EDL}} \varphi
$$

This thus provides a new axiomatization of DEL-validities. This new axiomatization is just made of $\Gamma\left(M^{d}\right)$ together with the axiomatization of EDL .

\section{Discussion and Conclusion}

We have presented an epistemic dynamic logic EDL whose semantics differs from that of Baltag et al.'s dynamic epistemic logic DEL. We have shown that DEL can be embedded into EDL. This result allows to conclude that EDL is an interesting alternative to Baltag et al.'s logic, that allows to talk about agents' perception of events just in the same way as DEL does. However, EDL is more expressive than DEL because it allows to talk about past events. Another of its advantages is that we can partly describe an event taking place and still draw inferences from this partial description, whereas in DEL the action (event) model has to specify everything. More generally, EDL seems more versatile than DEL to describe events. This allows to model some events that could not be modelled in DEL .

Let us demonstrate this last point by an example. Consider the situation where there are two agents $i$ and $j$, and there are two possible private announcements $a$ and $b$ with respective preconditions $p$ and $\neg p$. Suppose none of the agents knows anything beyond the mere fact that both $a$ and $b$ could have happened, i.e. $\left\langle a^{-}\right\rangle \top \vee\left\langle b^{-}\right\rangle \top$ is common knowledge. From this we should infer that the agents do not know anything about the other agent's perception (which is indeed true in reality). We can model this last fact as follows. First we recursively define the following set of formulas.

$$
\begin{aligned}
& -\Phi_{i}^{0}=\Phi_{j}^{0}=\left\{\left\langle a^{-}\right\rangle \top,\left\langle b^{-}\right\rangle \top\right\} \\
& -\Phi_{i}^{n}=\left\{B_{i} \varphi_{j}: \varphi_{j} \in \Phi_{j}^{n-1}\right\} \cup\left\{\bigwedge_{\left\{\varphi_{j}: \varphi_{j} \in \Phi_{j}^{n-1}\right\}} \hat{B}_{i} \varphi_{j}\right\}
\end{aligned}
$$

For example we have

$$
\begin{aligned}
\Phi_{i}^{1}= & \left\{B_{i}\left\langle a^{-}\right\rangle \top, B_{i}\left\langle b^{-}\right\rangle \top, \hat{B}_{i}\left\langle a^{-}\right\rangle \top \wedge \hat{B}_{i}\left\langle b^{-}\right\rangle \top\right\} \text { and } \\
\Phi_{j}^{2}= & \left\{B_{j} B_{i}\left\langle a^{-}\right\rangle \top, B_{j} B_{i}\left\langle b^{-}\right\rangle \top, B_{j}\left(\hat{B}_{i}\left\langle a^{-}\right\rangle \top \wedge \hat{B}_{i}\left\langle b^{-}\right\rangle \top\right)\right\} \cup \\
& \left\{\hat{B}_{j} B_{i}\left\langle a^{-}\right\rangle \top \wedge \hat{B}_{j} B_{i}\left\langle b^{-}\right\rangle \top \wedge \hat{B}_{j}\left(\hat{B}_{i}\left\langle a^{-}\right\rangle \top \wedge \hat{B}_{i}\left\langle b^{-}\right\rangle \top\right)\right\} .
\end{aligned}
$$

We naturally claim that the set of all $\left(\bigvee \Phi_{i}^{n}\right) \wedge\left(\bigvee \Phi_{j}^{n}\right)$ represents the fact that the agents do not know anything about the other agent's perception. Then we can prove by induction on $n$ that $\left\{\left\langle a^{-}\right\rangle \top \vee\left\langle b^{-}\right\rangle \top\right\} \vdash_{\mathrm{EDL}}\left(\bigvee \Phi_{i}^{n}\right) \wedge\left(\bigvee \Phi_{j}^{n}\right)$ for every 
$n .^{2}$ This indicates that the agents' incomplete knowledge of what is going on is correctly represented by $\left\{\left\langle a^{-}\right\rangle T \vee\left\langle b^{-}\right\rangle T\right\}$. Such situations cannot be described in DEL because this would require an infinity of atomic DEL-events, and the dynamic model $M^{d}$ would have to be infinite. So, in a sense, EDL seems to be more appropriate to represent situations where agents have only little cues about what is going on.

Another approach mapping DEL to automata propositional dynamic logic is [18]. He does not resort to converse events and translates dynamic models into a transformation on PDL programs. As we said in section 1, Yap introduced converse events into DEL but she failed to get a reduction axiom for the converse modal operator. Like us, she does not deal with belief revision and we leave the integration of belief revision mechanisms into EDL to further work. Another line of research is to study decidability and complexity of EDL .

\section{References}

1. Carlos Alchourrón, Peter Gärdenfors, and David Makinson. On the logic of theory change: Partial meet contraction and revision functions. J. of Symbolic Logic, 50:510-530, 1985 .

2. Alexandru Baltag. A logic of epistemic actions. Technical report, CWI, 2000. http://www. cwi.nl/ abaltag/papers.html.

3. Alexandru Baltag and Lawrence S. Moss. Logics for epistemic programs. Synthese, 139(2):165-224, 2004.

4. Alexandru Baltag, Lawrence S. Moss, and Slawomir Solecki. The logic of public announcements, common knowledge, and private suspicions. In Proc. TARK'98, pages 43-56. Morgan Kaufmann, 1998.

5. Ronald Fagin, Joseph Y. Halpern, Yoram Moses, and Moshe Y. Vardi. Reasoning about knowledge. MIT Press, 1995.

6. Jelle Gerbrandy. Bisimulations on Planet Kripke. PhD thesis, University of Amsterdam, 1999.

7. Jelle Gerbrandy and Willem Groeneveld. Reasoning about information change. J. of Logic, Language and Information, 6(2), 1997.

8. Andreas Herzig, Jérôme Lang, Dominique Longin, and Thomas Polacsek. A logic for planning under partial observability. In Proc. Nat. (US) Conf. on Artificial Intelligence (AAAI'2000), Austin, Texas, August 2000.

9. Hirofumi Katsuno and Alberto O. Mendelzon. On the difference between updating a knowledge base and revising it. In Peter Gärdenfors, editor, Belief revision, pages 183-203. Cambridge University Press, 1992. (preliminary version in Allen, J.A., Fikes, R., and Sandewall, E., eds., Principles of Knowledge Representation and Reasoning: Proc. 2nd Int. Conf., pages 387-394. Morgan Kaufmann Publishers, 1991).

10. J. A. Plaza. Logics of public communcations. In M. L. Emrich, M. Z. Pfeifer, M. Hadzikadic, and Z. W. Ras, editors, Proc. 4th Int. Symposium on Methodologies for Intelligent Systems, pages 201-216, 1989.

\footnotetext{
$\overline{2}$ The key observation is that $\vdash_{\mathrm{EDL}} B_{i}\left(\left\langle a^{-}\right\rangle \top \vee\left\langle b^{-}\right\rangle \top\right) \rightarrow\left(B_{i}\left\langle a^{-}\right\rangle \top \vee B_{i}\left\langle b^{-}\right\rangle \top \vee\right.$ $\left.\left(\hat{B}_{i}\left\langle a^{-}\right\rangle \top \wedge \hat{B}_{i}\left\langle b^{-}\right\rangle \top\right)\right)$
} 
11. H. Sahlqvist. Completeness and correspondence in the first and second order semantics for modal logics. In Stig Kanger, editor, Proc. 3rd Scandinavian Logic Symposium 1973, number 82 in Studies in Logic. North Holland, 1975.

12. Krister Segerberg. Belief revision from the point of view of doxastic logic. Bulletin of the IGPL, 3:534-553, 1995.

13. Krister Segerberg. Two traditions in the logic of belief: bringing them together. In Hans Jürgen Ohlbach and Uwe Reyle, editors, Logic, Language and Reasoning: essays in honour of Dov Gabbay, volume 5 of Trends in Logic, pages 135-147. Kluwer Academic Publishers, Dordrecht, 1999.

14. Johan van Benthem. One is a lonely number: on the logic of communication. In Z. Chatzidakis, P. Koepke, and W. Pohlers, editors, Logic Colloquium'02, pages 96-129. ASL \& A.K. Peters, Wellesley MA, 2006. Tech Report PP-2002-27, ILLC Amsterdam (2002).

15. Johan van Benthem and Eric Pacuit. The tree of knowledge in action: Towards a common perspective. In Advances in Modal Logic, pages 87-106, 2006.

16. Hans P. van Ditmarsch. Descriptions of game actions. J. of Logic, Language and Information (JoLLI), 11:349-365, 2002.

17. Hans P. van Ditmarsch, Wiebe van der Hoek, and Barteld Kooi. Dynamic Epistemic Logic. Kluwer Academic Publishers, 2007.

18. Jan van Eijck. Reducing dynamic epistemic logic to pdl by program transformation. Technical Report SEN-E0423, CWI, 2004.

19. Audrey Yap. Product update and looking backward. prepublications PP-2006-39, ILLC, 2006.

\section{A Proof of Proposition 2}

Proposition 4 (Proposition 2). Conditions (45), (nl), (nf) and (ed) are equivalent to conditions (45), $\left(n l^{i}\right),(n f)$ and (ed) where

$\left(n l^{i}\right) \quad \mathcal{B}_{i} \circ \mathcal{A}_{a}^{-1} \circ \mathcal{B}_{i} \circ \mathcal{A}_{a} \subseteq \mathcal{B}_{i}$

Proof. To prove $\left(\mathrm{nl}^{i}\right)$ from $(\mathrm{nl})$ and (ed), suppose $w_{1}\left(\mathcal{B}_{i} \circ \mathcal{A}_{b}^{-1} \circ \mathcal{B}_{i} \circ \mathcal{A}_{b}\right) w_{2}^{\prime}$, i.e. there are $w_{1}^{\prime}, v_{1}^{\prime}, v_{2}^{\prime}$ such that $w_{1} \mathcal{B}_{i} w_{1}^{\prime} \mathcal{A}_{b}^{-1} v_{1}^{\prime} \mathcal{B}_{i} v_{2}^{\prime} \mathcal{A}_{b} w_{2}^{\prime}$. We have to prove that $w_{1} \mathcal{B}_{i} w_{2}^{\prime}$. As we have $\left(\mathcal{A}_{b} \circ \mathcal{B}_{i} \circ \mathcal{A}_{b}^{-1}\right)\left(v_{1}^{\prime}\right) \neq \emptyset, v_{1}^{\prime} \mathcal{A}_{b} w_{1}^{\prime}$ and $v_{1}^{\prime}\left(\mathcal{B}_{i} \circ \mathcal{A}_{b}\right) w_{2}^{\prime}$, by (nl) we have $w_{1}^{\prime} \mathcal{B}_{i} w_{2}^{\prime}$. Then by transitivity of $\mathcal{B}_{i}$ we have $w_{1} \mathcal{B}_{i} w_{2}^{\prime}$.

To prove (nl) from $\left(\mathrm{nl}^{i}\right)$ and (ed), suppose $\left(\mathcal{A}_{a} \circ \mathcal{B}_{i} \circ \mathcal{A}_{b}^{-1}\right)(v) \neq \emptyset$, i.e. there are $w_{1}, w_{1}^{\prime}, v_{1}^{\prime}$ such that $v \mathcal{A}_{a} w_{1} \mathcal{B}_{i} w_{1}^{\prime} \mathcal{A}_{b}^{-1} v_{1}^{\prime}$. Moreover, suppose $v\left(\mathcal{B}_{i} \circ\right.$ $\left.\mathcal{A}_{b}\right) w_{2}^{\prime}$, i.e. there is $v_{2}^{\prime}$ such that $v \mathcal{B}_{i} v_{2}^{\prime} \mathcal{A}_{b} w_{2}^{\prime}$. We have to prove that for every $w_{2}$ such that $v \mathcal{A}_{a} w_{2}$ we also have $w_{2} \mathcal{B}_{i} w_{2}^{\prime}$. By (45) we have $v_{1}^{\prime} \mathcal{B}_{i} v_{2}^{\prime}$. We thus have $w_{1} \mathcal{B}_{i} w_{1}^{\prime} \mathcal{A}_{b}^{-1} v_{1}^{\prime} \mathcal{B}_{i} v_{2}^{\prime} \mathcal{A}_{b} w_{2}^{\prime}$. By $\left(\mathrm{nl}^{i}\right)$ we get $w_{1} \mathcal{B}_{i} w_{2}^{\prime}$. Finally, by (ed) we have $w_{2} \mathcal{B}_{i} w_{2}^{\prime}$, too.

\section{B Proof of Lemma 1}

Lemma 4 (Lemma 1). For every $\mathcal{L}_{D E L}$-formula $\varphi$ we have $\Gamma\left(M^{d}\right) \models_{E D L}\langle a\rangle \varphi \rightarrow$ $[a] \varphi$. 
Proof. Let $M^{d}=\left\langle E V T\right.$, Pre, $\left.\left\{\stackrel{d}{\longrightarrow}_{i}\right\}_{i \in A G T}\right\rangle$ be given, and let $M$ be an EDLmodel such that $M \models \psi$ for every $\psi \in \Gamma\left(M^{d}\right)$. Assume $w_{0} \mathcal{A}_{a} v_{0}$ and $w_{0} \mathcal{A}_{a} u_{0}$ with $v_{0} \neq u_{0}$. We are going to show that $u_{0}$ and $v_{0}$ are bisimilar.

$Z^{e}$ is defined to be an epistemic bisimulation between models $M_{1}$ and $M_{2}$ if $Z^{e}$ is a bisimulation between the restriction of these models to epistemic accessibility relations. Let $Z^{e}:=\{(w, w): w \in W\} \cup\left\{\left(v_{0}, u_{0}\right)\right\}$. We are going to show that $Z^{e}$ is an epistemic bisimulation. To do so, we need to prove

1. $u_{0} \in V(p)$ iff $v_{0} \in V(p)$ for all $p \in P R O P$;

2. if $v_{0} \mathcal{B}_{i} v^{\prime}$ then $u_{0} \mathcal{B}_{i} v^{\prime}$

3. if $u_{0} \mathcal{B}_{i} u^{\prime}$ then $v_{0} \mathcal{B}_{i} u^{\prime}$

(1) is guaranteed by Definition 1 (1). (2) and (3) are guaranteed by epistemic determinism: (ed) makes that $\mathcal{B}_{i}(u)=\mathcal{B}_{i}(v)$.

Now from $Z^{e}$, we are going to build up a bisimulation. We proceed as follows. $Z^{0}:=Z^{e}$

$Z^{n+1}:=\left\{\left(u_{n+1}, v_{n+1}\right): u_{n} \mathcal{A}_{a} u_{n+1}\right.$ and $v_{n} \mathcal{A}_{a} v_{n+1}$ for some $a \in E V T$ and $\left.u_{n} Z^{n} v_{n}\right\}$;

$Z:=\bigcup_{n \in \mathbb{N}} Z^{n}$.

We are going to show that $Z$ is a bisimulation.

1. We first show that $Z$ is an epistemic bisimulation.

We prove by induction on $n$ that every $Z^{n}$ is an epistemic bisimulation.

We have already proved that $Z^{0}$ is an epistemic bisimulation. Assume it is true for $Z^{n}$ and $u_{n+1} Z^{n+1} v_{n+1}$. Then there are $u_{n}, v_{n}$ such that $u_{n} Z^{n} v_{n}$, $u_{n} \mathcal{A}_{a} u_{n+1}$ and $v_{n} \mathcal{A}_{a} v_{n+1}$.

(a) $u_{n} \in V(p)$ iff $v_{n} \in V(p)$ because $Z^{n}$ is an epistemic bisimulation. So $u_{n+1} \in V(p)$ iff $v_{n+1} \in V(p)$ by Definition 1 (1).

(b) Assume $u_{n+1} \mathcal{B}_{i} u_{n+1}^{\prime}$. As by seriality of $\stackrel{d}{\longrightarrow}$ the set $\{b: a \stackrel{d}{\longrightarrow} b\}$ is nonempty, there is $b \in E V T, u_{n}^{\prime} \in W$ such that $u_{n}^{\prime} \mathcal{A}_{b} u_{n+1}^{\prime}$ by Definition $1(3)$.

Then $u_{n} \mathcal{B}_{i} u_{n}^{\prime}$ by (nf).

Then there is $v_{n}^{\prime} \in W$ such that $v_{n} \mathcal{B}_{i} v_{n}^{\prime}$ and $v_{n}^{\prime} Z^{n} u_{n}^{\prime}$ by induction hypothesis.

But $M, u_{n}^{\prime} \models \operatorname{Pre}(b)$ and besides for all $\varphi \in \mathcal{L}^{e}, M, v_{n}^{\prime} \models \varphi$ iff $M, u_{n}^{\prime} \models \varphi$ because $Z^{n}$ is an epistemic bisimulation by induction hypothesis.

So $M, v_{n}^{\prime} \models \operatorname{Pre}(b)$.

Then there is $v_{n+1}^{\prime}$ such that $v_{n}^{\prime} \mathcal{A}_{b} v_{n+1}^{\prime}$ by Definition 1 (2).

So $v_{n+1}^{\prime} \in \mathcal{B}_{i} \circ \mathcal{A}_{b}\left(v_{n}\right)$.

Besides $M, u_{n} \models \hat{B}_{i} \operatorname{Pre}(b)$, so $M, v_{n} \models \hat{B}_{i} \operatorname{Pre}(b)$ by induction hypothesis and because $\hat{B}_{i} \operatorname{Pre}(b) \in \mathcal{L}^{e}$.

So $M, v_{n}=[a] \hat{B}_{i}\left\langle b^{-}\right\rangle \top$ by Definition 1 (4).

But $M, v_{n} \models\langle a\rangle \top$, so $M, v_{n} \models\langle a\rangle \hat{B}_{i}\left\langle b^{-}\right\rangle \top$.

So $\left(\mathcal{A}_{a} \circ \mathcal{B}_{i} \circ \mathcal{A}_{b}^{-1}\right)\left(v_{n}\right) \neq \emptyset$.

So $\left(\mathcal{B}_{i} \circ \mathcal{A}_{b}\right)\left(v_{n}\right) \subseteq \mathcal{B}_{i}\left(v_{n+1}\right)$ by $(\mathrm{nl})$.

So $v_{n+1}^{\prime} \in \mathcal{B}_{i}\left(v_{n+1}\right)$ 
Besides $u_{n}^{\prime} Z^{n} v_{n}^{\prime}$ and $u_{n}^{\prime} \mathcal{A}_{b} u_{n+1}^{\prime}, v_{n}^{\prime} \mathcal{A}_{b} v_{n+1}^{\prime}$.

So by definition of $Z^{n+1}, u_{n+1}^{\prime} Z^{n+1} v_{n+1}^{\prime}$.

So there is $v_{n+1}^{\prime}$ such that $v_{n+1} \mathcal{B}_{i} v_{n+1}^{\prime}$ and $u_{n+1}^{\prime} Z^{n+1} v_{n+1}^{\prime}$

(c) The case $v_{n+1} \mathcal{B}_{i} v_{n+1}^{\prime}$ is similar.

So for all $n \in \mathbb{N}, Z^{n}$ is an epistemic bisimulation. Henceforth $Z$ is also a bisimulation.

2. Now we are going to show that $Z$ is a full bisimulation. Assume $u Z v$ for some $u, v \in W$. Then $u Z^{n} v$ for some $n \in \mathbb{N}$.

(a) If $u \mathcal{A}_{a} u^{\prime}$ then $M, u \models \operatorname{Pre}(a)$ by Definition 1 (2). So $M, v \models \operatorname{Pre}(a)$ because $Z$ is an epistemic bisimulation and $\operatorname{Pre}(a) \in \mathcal{L}^{e}$.

So there is $v^{\prime}$ such that $v \mathcal{A}_{a} v^{\prime}$. But then $u^{\prime} Z^{n+1} v^{\prime}$ by construction of $Z^{n}$. So $u^{\prime} Z v^{\prime}$.

(b) Similarly we prove that if $v \mathcal{A}_{a} v^{\prime}$ then there is $u^{\prime}$ such that $u \mathcal{A}_{a} u^{\prime}$ and $u^{\prime} Z v^{\prime}$.

\section{Proof of Lemma 2}

For any formula $\varphi$ we define the integer $\delta(\varphi)$ as the maximal number of nested event operator occurrences as follows:

$$
\begin{aligned}
& -\delta(p)=0 \\
& \left.-\delta\left(\varphi_{1} \wedge \varphi_{2}\right)=\max \left(\delta\left(\varphi_{1}\right), \delta\left(\varphi_{2}\right)\right)\right) \\
& -\delta(\neg \varphi)=\delta\left(B_{i} \varphi\right)=\delta(\varphi) \\
& -\delta([a] \varphi)=\delta\left(\left[a^{-}\right] \varphi\right)=\delta(\varphi)+1
\end{aligned}
$$

Lemma 5 (Lemma 2). Let $M^{d}$ be a DEL dynamic model, and let $\psi$ be a formula from $\mathcal{L}_{D E L}$. If $\nvdash_{D E L} \psi$ then $\Gamma\left(M^{d}\right) \nvdash_{E D L} \psi$.

Proof. We have to prove that if there is a static DEL-model $M^{s}$ and a $w$ in $M^{s}$ such that $M^{s}, w=\psi$ then $M^{s}$ can be turned into a EDL-model $M$ such that $M \models \Gamma\left(M^{d}\right)$, with a $w^{\prime}$ in $M$ such that $M, w^{\prime} \models \psi$.

The proof iteratively applies the product construction to the initial $M^{s}$ as follows: we set $M^{0}=M^{s}$, and where

$$
\begin{aligned}
& \quad M^{n+1}=M^{n} \otimes_{E D L} M^{d}=\left\langle W^{n+1}, V^{n+1},\left\{\mathcal{A}_{a}^{n+1}\right\}_{a \in E V T},\left\{\mathcal{B}_{i}^{n+1}\right\}_{i \in A G T}\right\rangle, \\
& \text { where } \\
& -W^{n+1}=W^{n} \cup\left\{\langle w, a\rangle: w \in W^{n} \text { and } M^{s}, w \models \operatorname{Pre}(a)\right\} ; \\
& -V^{n+1}(p)=V^{n}(p) \cup\left\{\langle w, a\rangle: w \in W^{n} \text { and } w \in V^{n}(p)\right\} ; \\
& -\mathcal{A}_{a}^{n+1}=\mathcal{A}_{a}^{n} \cup\left\{\langle w,\langle w, a\rangle\rangle: w \in W^{n}\right\} ; \\
& -\mathcal{B}_{i}^{n+1}=\mathcal{B}_{i}^{n} \cup\left\{\left\langle\left\langle w_{1}, a_{1}\right\rangle,\left\langle w_{2}, a_{2}\right\rangle\right\rangle: w_{1} \mathcal{B}_{i}^{n} w_{2} \text { and } a_{1} \stackrel{d}{\longrightarrow}{ }_{i} a_{2}\right\} .
\end{aligned}
$$

Note that we use $\otimes_{E D L}$ to distinguish our product construction here from DEL's. Finally, we set $M^{\infty}=\left\langle W^{\infty}, V^{\infty},\left\{\mathcal{A}_{a}^{\infty}\right\}_{a \in E V T},\left\{\mathcal{B}_{i}^{\infty}\right\}_{i \in A G T}\right\rangle$, where $W^{\infty}=$ $\bigcup_{n} W^{n}, V^{\infty}(p)=\bigcup_{n} V^{n}(p), \mathcal{A}_{a}^{\infty}=\bigcup_{n} \mathcal{A}_{a}^{n}$, and $\mathcal{B}_{i}^{\infty}=\bigcup_{n} \mathcal{B}_{i}^{n}$. Note that is just as Yap's construction [19]. We are going to prove that $M^{\infty}, w=\varphi$. Then we will show that $M^{\infty} \models \Gamma\left(M^{d}\right)$. First we prove a lemma: 
Lemma 6. Let $k \geq 0 .\left(M^{s} \otimes M^{d}\right)^{k},\langle w, a\rangle \leftrightarrow M^{k+1},\langle w, a\rangle$, where $\left(M^{s} \otimes M^{d}\right)^{k}$ is the result of the iteration process applied $k$ times to the static model $M^{s} \otimes M^{d}$ and the dynamic model $M^{d}$.

Proof. We prove it by induction on $k$.

$k=0:\left(M^{s} \otimes M^{d}\right)^{0}=M^{s} \otimes M^{d}$ and $M^{1}=M^{s} \otimes_{E D L} M^{d}$. Then by definition of $\otimes_{E D L}$, we clearly have $\left(M^{s} \otimes M^{d}\right)^{0},\langle w, a\rangle \leftrightarrows M^{1},\langle w, a\rangle$

$k+1:\left(M^{s} \otimes M^{d}\right)^{k+1}=\left(M^{s} \otimes M^{d}\right)^{k} \otimes_{E D L} M^{d}$. Now $\left(M^{s} \otimes M^{d}\right)^{k},\langle w, a\rangle \leftrightarrow M^{k+1},\langle w, a\rangle$

by induction hypothesis. So $\left(M^{s} \otimes M^{d}\right)^{k} \otimes_{E D L} M^{d},\langle w, a\rangle \leftrightarrow M^{k+1} \otimes_{E D L}$

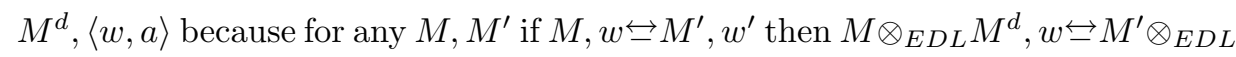
$M^{d}, w^{\prime}$.

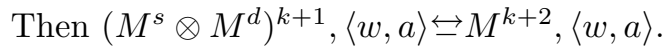

Now we prove a second lemma:

Lemma 7. For all $\varphi \in \mathcal{L}_{D E L}, M^{s}, w \models \varphi$ iff $M^{\infty}, w \models \varphi$

Proof. We set $\mathcal{P}(k)$ : "For all $\varphi \in \mathcal{L}_{\text {DEL }}$ such that $\delta(\varphi)=k, M^{s}, w \models \varphi$ iff $M^{k}, w \models \varphi$ ", where $M^{s}$ is the static model and $M^{k}$ is the iteration of the product construction.

We prove $\mathcal{P}(k)$ for all $k$ by induction on $k$.

$k=0$ : Then $\varphi$ is epistemic so it works by definition of $\otimes_{E D L}$.

$k+1$ : We prove it by induction on $\varphi$.

$-\varphi=[a] \varphi^{\prime}$.

$M^{s}, w \models[a] \varphi^{\prime}$

iff if $M^{s}, w \models \operatorname{Pre}(a)$ then $M^{s} \otimes M^{d},\langle w, a\rangle \models \varphi^{\prime}$

iff if $M^{s}, w \models \operatorname{Pre}(a)$ then $\left(M^{s} \otimes M^{d}\right)^{k},\langle w, a\rangle \models \varphi^{\prime}$ by Induction

Hypothesis because $\delta\left(\varphi^{\prime}\right) \leq k$,

iff if $M^{s}, w \models \operatorname{Pre}(a)$ then $M^{k+1},\langle w, a\rangle \models \varphi^{\prime}$ by Lemma 6

iff if $M^{k+1}, w \models \operatorname{Pre}(a)$ then $M^{k+1},\langle w, a\rangle \mid=\varphi^{\prime}$

iff $M^{k+1}, w \models[a] \varphi^{\prime}$ by definition of $\otimes_{E D L}$

iff $M^{k+1}, w \models \varphi$.

$-\varphi=\varphi_{1} \wedge \varphi_{2}$ works by Induction Hypothesis.

$-\varphi=B_{i} \varphi^{\prime}$ works as well.

$-\varphi=p$ is impossible because $k+1 \geq 1$.

Then we can easily prove that for all $\varphi$ such that $\delta(\varphi)=k, M^{k}, w \models \varphi$ iff $M^{\infty}, w \models \varphi$. Then for all $k$, for all $\varphi$ such that $\delta(\varphi)=k, M^{s}, w \models \varphi$ iff $M^{\infty}, w \models \varphi$

i.e. for all $\varphi \in \mathcal{L}_{\mathrm{DEL}}, M^{s}, w \models \varphi$ iff $M^{\infty}, w \models \varphi$. In particular, because $M^{s}, w \models \psi$, we have $M^{\infty}, w \models \psi$.

It remains to prove that $M^{\infty} \models \Gamma\left(M^{d}\right)$. Conditions (1) and (2) of definition 5.1 are clearly fulfilled. As for condition (3), let $w \in W^{\infty}, w^{\prime}$ is such that 
$w \mathcal{A}_{a} w^{\prime}$ iff $w^{\prime}=\langle w, a\rangle$. Now $\langle w, a\rangle \mathcal{B}_{i} u$ iff $u=\langle v, b\rangle$ with $w \mathcal{B}_{i} v$ and $a \stackrel{d}{\longrightarrow}{ }_{i} b$ by definition of $\otimes_{E D L}$. So for all $u$ such that $\langle w, a\rangle \mathcal{B}_{i} u$, there is $b$ and $v$ such that $a \stackrel{d}{\longrightarrow} b$ and $v \mathcal{A}_{b} u$. This proves that $M^{\infty}, w=[a] B_{i}\left(\left\langle b_{1}^{-}\right\rangle \top \vee \ldots \vee\left\langle b_{n}^{-}\right\rangle \top\right)$ where $b_{1}, \ldots, b_{n}$ is the list of all $b$ such that $a \stackrel{d}{\longrightarrow} b$. Finally, concerning condition (4), assume $M^{\infty}, w \models \hat{B}_{i} \operatorname{Pre}(b)$ and $w \mathcal{A}_{a}\langle w, a\rangle$. Then there is $v$ such that $w \mathcal{B}_{i} v$ and $v \mathcal{A}_{b}\langle v, b\rangle$. So by definition of $\otimes_{E D L}$, because $a \stackrel{d}{\longrightarrow} i b$, we have $\langle w, a\rangle \mathcal{B}_{i}\langle v, b\rangle$. Hence $M^{\infty},\langle w, a\rangle \models \hat{B}_{i}\left\langle b^{-}\right\rangle \top$ and finally $M^{\infty}, w \models[a] \hat{B}_{i}\left\langle b^{-}\right\rangle \top$.

\section{Proof of Lemma 3}

Lemma 8 (Lemma 3). Let $M^{d}$ be a DEL dynamic model, and let $\psi$ be a formula from $\mathcal{L}_{D E L}$. If $\models_{D E L} \psi$ then $\Gamma\left(M^{d}\right) \models_{E D L} \psi$.

Proof. We take advantage of the complete axiomatization of DEL-validities given in $[4,3]$, and show that the DEL-axioms are EDL-valid, and that the DELinference rules preserve EDL-validity. As the inference rules of DEL and EDL are identical (viz. modus ponens and necessitation) it is clear that the DELinference rules preserve EDL-theoremhood. It is straightforward to show that every instance of the DEL-axioms not involving dynamic operators is EDL-valid. So what remains is to prove that the schemas

$(\mathrm{A} 1)[a] p \leftrightarrow(\operatorname{Pre}(a) \rightarrow p)$

(A2) $[a] \neg \varphi \leftrightarrow(\operatorname{Pre}(a) \rightarrow \neg[a] \varphi)$

(A3) $[a] B_{i} \varphi \leftrightarrow\left(\operatorname{Pre}(a) \rightarrow B_{i}\left[b_{1}\right] \varphi \wedge \ldots \wedge B_{i}\left[b_{n}\right] \varphi\right)$

where $b_{1}, \ldots, b_{n}$ is the list of all $b$ such that $a \stackrel{d}{\longrightarrow}$, are logical consequences of $\Gamma\left(M^{d}\right)$ in EDL.

(A1) Axiom (A1) can be proved from the nonlogical axioms 1 (1) $p \rightarrow[a] p$ and $1(2)\langle a\rangle \top \leftrightarrow \operatorname{Pre}(a)$ of the theory $\Gamma\left(M^{d}\right)$.

(A2) For the left-to-right direction of (A2) we have

$$
\Gamma\left(M^{d}\right) \models_{\mathrm{EDL}}([a] \neg \varphi \wedge \operatorname{Pr}(a) \wedge[a] \varphi) \rightarrow \perp
$$

because of the nonlogical axiom $1(2)\langle a\rangle \top \leftrightarrow \operatorname{Pre}(a)$ of $\Gamma\left(M^{d}\right)$.

For the right-to-left direction, on the one hand we have $\Gamma\left(M^{d}\right) \models_{\text {EDL }}$ $\neg \operatorname{Pre}(a) \rightarrow[a] \perp$ again by the nonlogical axiom $1(2)$, and on the other hand $\Gamma\left(M^{d}\right) \models$ EDL $\neg[a] \varphi \rightarrow[a] \neg \varphi$ by Lemma 1.

(A3) For the left-to-right direction of (A3), suppose

$$
M, w \models[a] B_{i} \varphi \wedge \operatorname{Pre}(a),
$$

and suppose $M, w \models \neg B_{i}[b] \varphi$ for some $b$ such that $a \stackrel{d}{\longrightarrow} i b$. So there must exist worlds $w^{\prime}$ and $v^{\prime}$ such that $w \mathcal{B}_{i} w^{\prime}, w^{\prime} \mathcal{A}_{b} v^{\prime}$ and $M, v^{\prime} \models \neg \varphi$. Therefore $M, w^{\prime} \models \operatorname{Pre}(b)$ by nonlogical axiom 1 (2), and $M, w \models \hat{B}_{i} \operatorname{Pre}(b)$. As $\langle a, b\rangle \in \stackrel{d}{\longrightarrow}$, our nonlogical axiom 1 (4) tells us that $M, w=\hat{B}_{i} \operatorname{Pre}(b) \rightarrow$ $[a] \hat{B}_{i}\left\langle b^{-}\right\rangle \top$, and hence $M, w \models[a] \hat{B}_{i}\left\langle b^{-}\right\rangle \top$. As by hypothesis $M, w \models$ $\operatorname{Pre}(a)$, by nonlogical axiom $1(2)\left(\mathcal{A}_{a} \circ \mathcal{B}_{i} \circ \mathcal{A}_{b}^{-1}\right)(w) \neq \emptyset$. By the constraint (nl) on EDL-models we have 


$$
\left(\mathcal{B}_{i} \circ \mathcal{A}_{b}\right)(w) \subseteq\left(\mathcal{A}_{a} \circ \mathcal{B}_{i}\right)(w),
$$

i.e. $v^{\prime} \in\left(\mathcal{A}_{a} \circ \mathcal{B}_{i}\right)(w)$. As we have supposed that $M, w=[a] B_{i} \varphi$, we must have $M, v^{\prime} \models \varphi$, which is contradictory.

For the right-to-left direction of (A3), we know that $\Gamma\left(M^{d}\right) \models_{\mathrm{EDL}} \neg \operatorname{Pre}(a) \rightarrow$ $[a] \perp$ again by the nonlogical axiom $1(2)$, so it remains to prove that

$$
\Gamma\left(M^{d}\right)==_{\mathrm{EDL}}\left(B_{i}\left[b_{1}\right] \varphi \wedge \ldots \wedge B_{i}\left[b_{n}\right] \varphi\right) \rightarrow[a] B_{i} \varphi .
$$

where $b_{1}, \ldots, b_{n}$ is the list of all $b$ such that $a \stackrel{d}{\longrightarrow} b$.

Suppose $M, w \models B_{i}\left[b_{1}\right] \varphi \wedge \ldots \wedge B_{i}\left[b_{n}\right] \varphi$, and suppose $M, w \models \neg[a] B_{i} \varphi$. The latter implies that there are worlds $v$ and $v^{\prime}$ such that $w \mathcal{A}_{a} v \mathcal{B}_{i} v^{\prime}$ and $M, v^{\prime}=\neg \varphi$. By the nonlogical axiom 1 (3) we have

$$
[a] B_{i}\left(\left\langle b_{1}^{-}\right\rangle \top \vee \ldots \vee\left\langle b_{n}^{-}\right\rangle \top\right)
$$

where $b_{1}, \ldots, b_{n}$ is the list of all $b$ such that $a \stackrel{d}{\longrightarrow}{ }_{i} b$.

Hence $M, v^{\prime} \models\left\langle b_{1}^{-}\right\rangle \top \vee \ldots \vee\left\langle b_{n}^{-}\right\rangle \top$. By seriality of $\stackrel{d}{\longrightarrow}_{i}$ the set $\{b: a \stackrel{d}{\longrightarrow} i$ $b\}$ is nonempty. We therefore get that $M, v^{\prime} \models\left\langle b^{-}\right\rangle \top$ for some $b$ such that $a \stackrel{d}{\longrightarrow}$ b: there is some $w^{\prime}$ such that $w^{\prime} \mathcal{A}_{b} v^{\prime}$. Now by (nf) we must have $w \mathcal{B}_{i} w^{\prime}$. As $M, w=B_{i}[b] \varphi$ we must have $M, w^{\prime}=[b] \varphi$, and thus $M, v^{\prime} \models \varphi$, which is contradictory. 